\title{
Black Holes or objects without event horizon?
}

\author{
Leonid Verozub
}

December 6, 2021

Kharkiv University, Kharkiv, 61022, Kharkiv, UA

\begin{abstract}
In this paper, we investigate the question of whether the generally accepted interpretation of the supermassive object in M87, investigated by EHT collaboration, is the only possible one. There are grounds for this, in particular, due to the detection of a magnetic field in its vicinity. For this purpose, the stability of self-gravitating objects is investigated based on the correct definition of the energy of gravitation in the framework of the bi-metric approach to the theory.
\end{abstract}

Keywords: Black Holes; Theory of gravitation

\section{Introduction}

In work[1], bi-metric equations of gravitation were considered, according to which the gravitation of a point mass does not have a singularity. The force of gravity acting on a test particle at rest is

$$
F=\frac{m M G}{r^{2}}\left(1-\frac{r_{g}}{f}\right)
$$

where $r_{g}=2 G M / c^{2}$ is the Schwarzsschild radius of mass $\mathrm{M}, r$ is distance from the center, and $f=\left(r_{g}^{3}+r^{3}\right)^{1 / 3}$.

Singularity is missing in this model. The gravitational force decreases within the Schwarzschild radius, and eventually tends to zero along with the distance from the center.

Typically, the Schwarzschild radius is small compared with the dimensions of celestial objects. That is, almost always the condition $r \gg r_{g}$ is executed and therefore $F$ coincides with Newton's law.

However, this solution leads to the possibility of the existence of supermassive objects without an event horizon. The possibility of this was considered even before such an object was discovered in the center of our galaxy [2]. In 


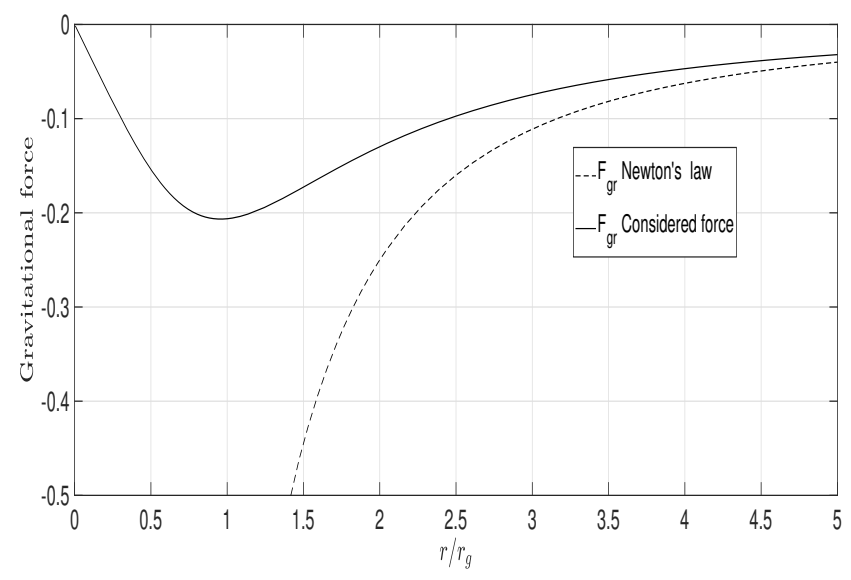

Figure 1: Gravitational force

paper [3] it was proposed as an alternative to black holes. Later, this possibility was studied in more detail in $[13,2, ?, 14]$. However, the nature of such objects remained unclear.

Here new result is presented in viewed on EHT $[4,5]$ collaboration results.

\section{Energy of gravitation}

A correct expression for the density of the gravitational field in the bi-metric model can be obtained directly from the expression for the force (1) in Minkowski space.

We define the potential of the force $F$ as $U(r)=\int_{0}^{r} \frac{F\left(r^{\prime}\right)}{m} d r^{\prime}$.

Double differentiation of function $U(r)$ gives

$$
U^{\prime \prime}=-\frac{2 U^{\prime}}{r}-\frac{2 G^{2} M^{2}}{c^{2} f^{4}} .
$$

Now denote

$$
t_{00}=\frac{G M^{2}}{2 \pi f^{4}}
$$

As a result, we obtain that function $U(r)$ satisfies the differential equation

$$
\nabla^{2} U=-4 \pi \bar{\rho}
$$

where the mass density is $\bar{\rho}=t_{00} / c^{2}$.

This equation has a clear physical meaning. This is the Poisson equation for a spherically symmetric field, in which $\bar{\rho}$ is the mass density of the gravitational field created by the mass $M$, since in the absence of another matter only the 
density of the gravitational field can lead to the fact that the potential will differ from zero.

We can also write

$$
t_{00}=\varkappa^{-1} \frac{r_{g}^{2}}{2 f^{4}}
$$

where $\varkappa=4 \pi G / c^{4}$ which gives, when integrating over entire space

$$
\int t_{00} d V=M c^{2}
$$

Thus the energy of a point mass is finite and equal to the expected value in accordance with the law of equivalence of mass and energy. In classical electrodynamics, a similar integral for the Coulomb field diverges.

For the above reasons, we can consider the expression (3) as the energy density of the gravitational field of a mass $M$ and use this fact here.

\section{Possible nature of the supermassive objects}

Consider the graph of the total energy $E$ of a spherically symmetric, selfgravitating configuration of a degenerate Fermi gas

Using the average values for the characteristics of the quantities used, $E$ can be written in the following form

$$
E=E_{k}-V t_{00} .
$$

where $E_{k}$ is the internal inergy of the $M_{0}$ formed by $\mathrm{N}$ protons in the volume $V=4 / 3 \pi R^{3}$. The second term $V t_{00}$ is the gravitational binding energy in volume $V$. Here

$$
t_{00}=\frac{G M_{0}^{2}}{2 \pi f^{4}},
$$

$f=\left(R^{3}+r_{g}^{3}\right)^{1 / 3}$ and $r_{g}=2 G M_{0} / c^{2}$.

These graph are shown in fig. 2 for several values of mass $M_{0}$. 

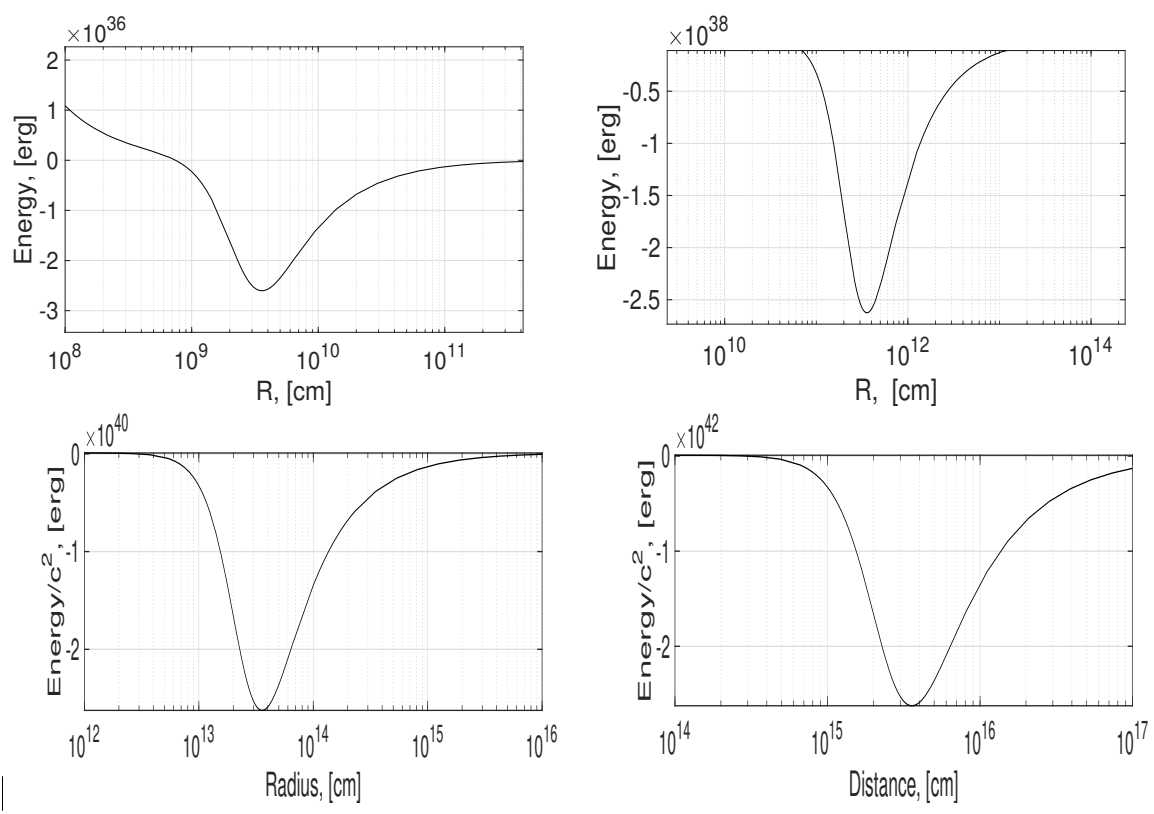

Figure 2: The mass $M=\mathcal{E} / c^{2}$ of the configuration as a function of total number of particles .

In equation $4, E_{k}$ is equal[10]:

$$
\begin{aligned}
& E_{k}=\frac{c V\left(m_{e} c\right)^{4}}{8 \pi^{2} \hbar^{3}}\left(x_{0}\left(2 x_{0}^{2}+1\right)\left(x_{0}^{2}+1\right)^{1 / 2}-\operatorname{asinh}\left(x_{0}\right) ;\right. \\
& p_{0}=\hbar\left(3 \pi^{2} n\right)^{1 / 3} \\
& x_{0}=\frac{p_{0}}{m_{e} c}, n=N / V
\end{aligned}
$$

$N$ is the particles number in volume $V$.

$M_{0}=N m_{n}, m_{e}$ and $m_{n}$ are mass of electron and neutron, respectively.

These graphs show that all configurations of self-gravitating objects are stable. The graphs also show that the mass of the completely degenerate Fermi gas not exceeding $10^{39} \mathrm{~g}$. Apparently, objects with large masses have a more complex structure, their outer layers have a low density.

It is also impossible to exclude gross errors in determining the mass of such objects[17]. However, in any case, one might think that objects with masses less than $10^{39} \mathrm{~g}$ are a viable alternative to black holes.

\section{Light deflection}

Let's find the radius of the ring of light from the object in M87. This can be done in two ways. First, the deflection angle, defined as the angle between the asymptotic incoming and outgoing trajectories, obtained in [6] for an arbitrary Lagrangian like used in [15] based on some Weinberg method. 


$$
\Theta=\arcsin \left(u \frac{C\left(r_{m}\right)}{B\left(r_{m}\right)}\right)^{1 / 2}
$$

where () $C=1-r_{g} / f, r_{g}=2 G M / c^{2}, M=E / c^{2}, f=\left(r_{g}^{3}+r^{3}\right)^{1 / 3}, r$ is the distance from the object center, $B=f^{2}, r_{g}$ is the closest approach distance of the light, $\mathrm{u}$ is the impact parameter, defined as the distance between the black hole and each of the asymptotic photon trajectories, related to $r_{m}$ by

$$
u=\left(\frac{B\left(r_{m}\right)}{C\left(r_{m}\right)}\right)^{1 / 2}
$$

Second, we can use Nemirov's simple and elegant method [7], suitable for flat space. This gives

$$
\Theta=\arcsin \left(\frac{R}{D}\left(\frac{1-r_{g} / D}{1-r_{g} / R}\right)^{1 / 2}\right)
$$

In this equation $R$ is the radius of the object, $r_{g}$ is its Schwarzschild radius, $D$ is the distance from an observer.

The both method gives the same result - 0.22 uuas that is consistent with observation [4]. However, a quantitative comparison is not possible for the result [5] due to the strong blurring of the inner boundary of the photon ring.

In addition, it should be noted that the existence of a magnetic field in the vicinity of the object is difficult to reconcile with the generally accepted interpretation of the M87 object as a Black Hole.

Thus, in fact, we do not have a quantitative test for a confident conclusion about the nature of M87.

There is, however, another method for this, which is discussed in the following sections.

\section{Schwarzschild radius of the visible Universe}

\subsection{Motion of a fluid}

The expanding Universe can be viewed as an isentropic fluid. It is shown in [15] that the macoscopically small elements ("particles") of such a spherically symmetric fluid are described in exact accordance with the relativistic Euler equations by the following Lagrangian

$$
L=-m c\left(G_{\alpha \beta} \frac{d x^{\alpha}}{d t} \frac{d x^{\beta}}{d t}\right)^{1 / 2}
$$

where $G_{\alpha \beta}=\chi^{2} g_{\alpha \beta}, \eta_{\alpha \beta}$ is a solution of our vacuum equations of gravitation in Minkowski space, $\chi=w / \rho c^{2}$ where $w$ is the volume density of the enthalpy

where

$$
\chi=\frac{w}{\rho c^{2}}=1-\frac{t_{00}}{\rho c^{2}}+\frac{p}{\rho c^{2}},
$$


where $w$ is the enthalpy per unit volume, $\rho$ is the density, $t_{00}$ is the density of gravitational binding energy for mass $M, p$ is the pressure.

The integral of motion is

$$
\frac{m c^{2} \chi}{[1-(\dot{r} / c)]^{1 / 2}}=\text { Const }
$$

This equation is the relativistic Bernoulli equation in Minkowski space.

The constant has a meaning of the energy of a particle and, therefore, this equation can be read as

$$
\frac{\chi}{\left(1-v^{2} / c^{2}\right)^{1 / 2}}=\bar{E}
$$

where the constant $\bar{E}=E / m c^{2}$ is the dimensionless energy $E$ of the "particles" of the fluid and $v=\dot{x}$.

At present, the pressure is negligible and therefore $\chi=1+\epsilon / \rho c^{2}$, and for small distance from an observer and at $\bar{E}=1$, the velocity $v(r)$ is proportional to the distance $r$ from the observer:

$$
v=\frac{4}{3} \sqrt{G \pi \rho} r
$$

We obtain the Hubble law holds and, at the density $\rho=6 \cdot 10^{-30} \mathrm{~g} \mathrm{~cm}^{-3}$, the Hubble constant is

$$
H_{0}=\frac{4}{3}(G \pi \rho)^{1 / 2}=1.5 \cdot 10^{-18} s^{-1}
$$

If $\bar{E}$ is not equal to 1 , the equations of fluid motion contradict the Hubble law.

\subsection{Acceleration of Universe as a test gravity properties}

The Lagrange equation obtained from $(7)$ is

$$
\ddot{r}=-c^{2} \frac{\chi^{\prime}(r)}{\chi(r)}\left(1-\frac{\dot{r}^{2}}{c^{2}}\right)
$$

which with the equation $v^{2} / c^{2}=1-\chi$ leads to the following equation for acceleration of a fluid in the expanding Universe.

$$
\ddot{r}=-c^{2} \frac{d \chi}{d r} \chi
$$

A graph of $\ddot{r}(r)$ is shown on the fig.3

This graph shows that the acceleration of distant particles rapidly tends to zero at distances to observed objects in excess of the value $R_{g}=c\left(\frac{3}{8 \pi G \rho}\right)^{1 / 2}$ were $\rho$ is the density of the observed Universe because $R_{g}=1.6 \cdot 10^{28} \mathrm{~cm}$ at $\rho=$ $6 \cdot 10^{-30} \mathrm{~g} / \mathrm{cm}^{3}$. 


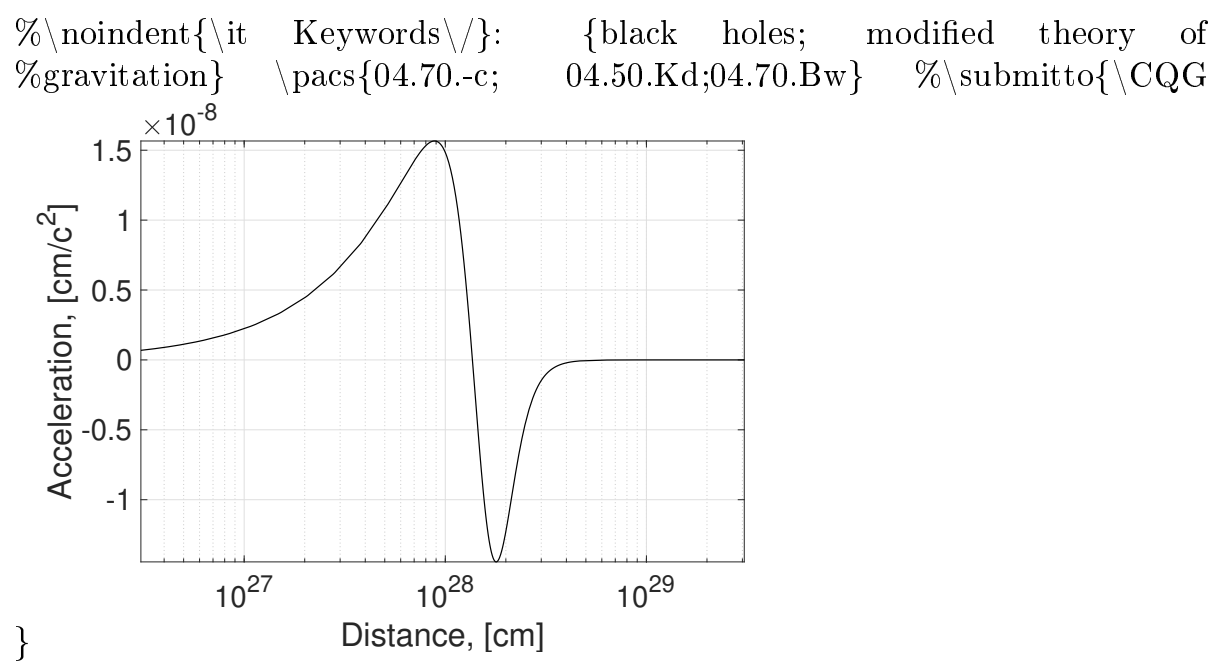

Figure 3: Distance from an observer vs. the Universe acceleration

The same should be said about the force of gravity, which is the acceleration of a unit mass.

Therefore, this value $R_{g}$ can be called the gravitational radius of the visible Universe.

At any point in the infinite universe, only gravity within the radius $R_{g}$ affects the observed matter.

The graph shows that the acceleration of the expansion of the universe, which cannot explain general relativity, is a consequence of the behavior of gravitation near the Schwarzschild radius $R_{g}$ of the Universe.

The reason for the acceleration of the expansion of the Universe is that its observable size approaches the Schwarzschild radius $R_{g}$ of the Universe.

This is a measurable quantity. And it is this that makes it possible to test the conclusion about the existence of black holes and the existence of a singularity.

For example, you can find the deceleration parameter:

$$
q=-\frac{\ddot{r} r}{\dot{r}^{2}} .
$$

gives some confidence in the above result.

The observation gives the present-day value of this parameter $q_{0}$ as approximately -0.55 . Graph 4 shows $q$ as a function of distance from the observer in the theory used here.

Graph 4 shows that the observed value of pre sent-day $q_{0} \approx-0.55$ corresponds to the geometrical distance of the remote objects of about $10^{28} \mathrm{~cm}$. It is consistent with fig. 3 that shows the acceleration of distance object. 


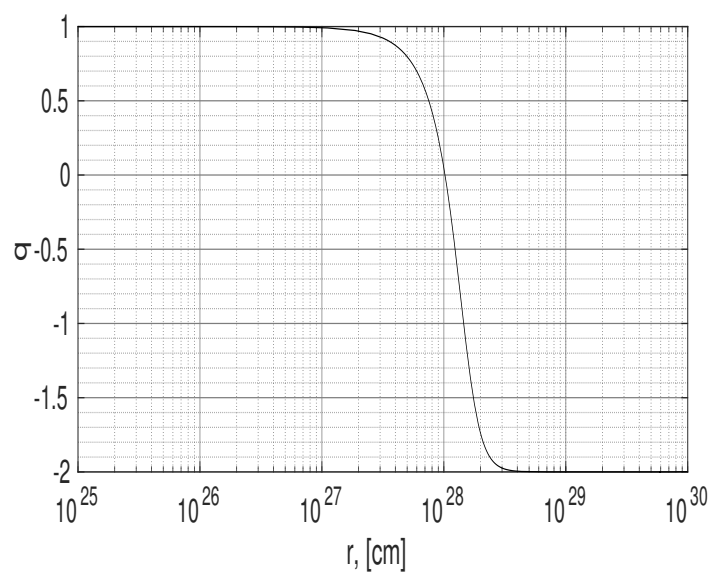

Figure 4: Parameter $q$ vs. distance to the object.

\section{Appendix}

\section{Physical meaning of the bimetricity}

In paper [1], the opinion was substantiated, which goes back to Rosen [8], that the theory of gravitation should be bi-metric. However, this assumption needs serious substantiation. The book [11] is devoted to this, and below is a brief substantiation of the description of gravitation in the Mikowski space.

In Einstein's classical theory, coordinates play a twofold and difficultly compatible role. On the one hand, coordinates are only a way to parameterize events, that is, points in space-time. From this point of view, they are completely arbitrary. On the other hand, they play the role of gauge transformations.

Rosen [8] was the first to recognize the need for introducing Minkowski space into theory. The possibility of considering Einstein's equations in flat space was also considered by some authors after the paper of Tirring[9].

The physical meaning of bimetrism used in the present paper is based on ideas going back to Poincaré, who realized that there is a strange situation: In order to characterize the properties of the geometry of space, we must know the properties of measuring instruments, and in order to characterize the properties of instruments, we must know the geometric space properties. In the modern interpretation, this can be summarized as follows: The physical, operational sense has only the aggregate "space-time geometry + properties of measuring instruments" [11]. In this form, this fact has never been realized in physics.

However, a step in its implementation can be made if we notice that it is the reference frame used by the observer is the measuring tool that is necessary to establish the geometric properties of space-time. Therefore, it should be assumed that the following statement is true: Only the combination "space- 
time geometry + properties of the reference frame used" has physical meaning.

Of course, by reference frame we mean here not a coordinate system, but a physical device consisting of a reference body and a clock attached to it.

Remembering all the above, we now consider a classical field $\mathcal{F}$ in an inertial reference system $(I R F)$, where space-time according to experience is Minkowski space. The world lines of the particles of mass $m$ moving under the action of the field $\mathcal{F}$ form the reference body of a non-inertial reference which can be named the proper reference frame $(P R F)$ of the field $\mathcal{F}$.

If an observer in a $P R F$ of the field $\mathcal{F}$ is at rest, his world line coincides with the world line of some point of the reference body. It is obvious for the observer that the accelerations of the point masses forming his reference body are equal to zero in non-relativistic and relativistic meaning. That is, if the line element of space-time in an inertial reference frame is denoted by $d \sigma$ and $u^{\alpha}=d x^{\alpha} / d \sigma$ is the field of 4 - velocities of the point masses forming the reference body, then the absolute derivative of $u^{\alpha}$ is equal to zero: ${ }^{1}$

$$
D u^{\alpha} / d \sigma=0
$$

(We mean that an arbitrary coordinate system is used.)

The same should occur in the PRF used. That is, if the line element of space-time in the $P F R$ is denoted by $d s$, the 4 -velocity vector $\zeta^{\alpha}=d x^{\alpha} / d s$ of the point-masses forming the reference body of the $P R F$ should satisfy the equation

$$
D \zeta^{\alpha} / d s=0
$$

The equation (10) uniquely determines the fundamental metric form in PRFs.

Indeed, the differential equations of these world lines are at the same time the Lagrange equations describing, in Minkowski space, the motion of the point masses forming the reference bodies of the $P R F$. The eq. (10) can be derived from a Lagrange action $S$ by the principle of the least action. Therefore, the equations of the geodesic lines can be obtained from a line element $d s=k d S$, where $k$ is a constant, $d S=\mathcal{L}(x, \dot{x}) d t$, and $\mathcal{L}(x, \dot{x})$ is a Lagrange function describing, in Minkowski space, the motion of identical point masses $m$ forming the body reference of the $P R F$. The constant $k$ is equal to $-(m c)^{-1}$, as follows from the analysis of the case when the frame of reference is inertial, when $\mathcal{L}(x, \dot{x})=-m c d s$ at the signature $(+---)$.

Thus, if we proceed from relativity of space and time in the Berkeley-LeibnizMach-Poincaré (BLMP) sense, then the line element of space-time in PRFs can be expected to have the following form [1] o

$$
d s=-(m c)^{-1} d S(x, d x) .
$$

For example, if the field $\mathcal{F}$ is electromagnetic, then the space-time in such reference frames is Finslerian[1, 11]. And the space-time in the reference frames comoving to an isentropic ideal fluid is conformal to the Minkowski space.

\footnotetext{
[16].

${ }^{1}$ We use notations and definitions, following the Landau and Lifshitz book
} 
In the case of gravity, we proceed from Thirring's assumption that gravity is described by a tesor field $\psi_{\alpha \beta}(x)$ and the Lagrangian describing the motion of test particles has the form

$$
L=-m c\left[g_{\alpha \beta}(\psi) \dot{x}^{\alpha} \dot{x}^{\beta}\right]^{1 / 2} .
$$

Then it is obvious that space-time in the PRF is Riemannian with a linear element of the form

$$
d s=\left(g_{\alpha \beta}(x) d x^{\alpha} d^{\beta}\right)^{1 / 2}
$$

This conclusion leads to the possibility of a double interpretation of gravity. Gravity can be considered as physical field $\psi$ in inertial reference frames and manifests itself as curvature in its own reference frames (PRFs) of this field. ${ }^{2}$

Possible equations of gravitation for the function $\psi(x)$ are considered in [11].

In the present paper, the description of gravity in Minkowski space is used since in this case we can get a correct expression for the field energy.

\section{References}

[1] L. Verozub, Annalen der Physik, 16, No. 1, p. 28, (2008)

[2] L. Verozub, Astronomische Nachrichten, 327, No. 4, p. 355, (2006)

[3] L.Verozub, E.Bannikova,arXiv: fstro-ph/9805299 (1998)

[4] EHT collaboration, Astroph.J.Lett., 875:L1 (2018)

[5] EHT collaboration, Astroph.J.Lett., 910:L12 (2021)

[6] V. Bozza, Gen.Relat. and Grav,42,2269 (2010)

[7] R. Nemiroff, Am.Journ. Phys., 61,619 (1993)

[8] N. Rosen, Gen. Relat. Grav., 4, 435 (1973)

[9] W. Thirring, Ann. Phys., 16, 96 (1961)

[10] L. Landau, E.Lifshitz, Statistic Physics, Elsevier (2013)

[11] L. Verozub, Space-time relativity and gravitation, Lambert Acad. Publ. (2017)

2 1) The question: "Where is this inertial reference frame ?" does not make sense, since, as noted in the footnote on page 1 , the properties of the reference system do not have a physical meaning in themselves. In the concrete, we can imagine that we are in an inertial reference frame and consider the gravity of the Universe as a physical field in Minkowski space, but we can also assume that we are in the reference frame comoving to the radial flow of galaxies and consider space-time as Riemannian.

2) Are both descriptions of gravity fully equivalent or just locally? This question remains open, but this plays no role for problem under consideration. 
[12] L. Verozub, Astron. Nachrichten 317, 107 (1996).

[13] L. Verozub and A. Kochetov, Astron. Nachrichten 322, 143 (2001).

[14] L. Verozub, Nuovo Cimento,123,1653 (2008).

[15] L. Verozub, Int. J.Mod.Phys.D, 17, 337 (2008)

[16] Landau, L.; Lifshitz, E. , The Classical Theory of Fields , 4th. Revised ed.; Pergamon, Oxford.

[17] L.,Titarchk et al, A\&A,633,A73 (2020) 